\title{
LAS REACCIONES POSTRAUMÁTICAS EN LA INFANCIA Y ADOLESCENCIA MALTRATADA: EL TRAUMA COMPLEJO
}

\author{
CONCEPCIÓN LÓPEZ-SOLER \\ Facultad de Psicología, Universidad de Murcia \\ Hospital Universitario Virgen Arrixaca, El Palmar, Murcia
}

\begin{abstract}
Resumen: Los menores expuestos a condiciones estresantes graves, tales como negligencia, abuso emocional y/o físico en las relaciones familiares primarias, pueden desarrollar traumas cuyos síntomas no se incluyen en el trastorno por estrés postraumático (TEPT). Las consecuencias del trauma complejo afectan el desarrollo psicológico, y las características se diagnostican en la actualidad en la categoría de trastorno por estrés postraumático extremo no especificado (disorders of extreme not otherwise specified, DESNOS) (Pelcovitz et al., 1992), cuyas alteraciones afectan a: a) regulación de los afectos e impulsos, b) memoria y atención; c) autopercepción, d) relaciones interpersonales, e) somatizaciones, y f) sistema de significados. Estos síntomas se incluyen en la propuesta de una nueva categoría diagnóstica: el trastorno por estrés postraumático complejo o extremo. Las características específicas de este trastorno en menores que presentan trauma complejo están siendo estudiadas (Cook et al., 2005). Se aportan datos sobre sintomatología del trauma complejo en menores maltratados.
\end{abstract}

Palabras clave: reacciones postraumáticas, TEPT, trauma complejo, DESNOS, maltrato infantil.

\section{Maltreatment of children and adolescentes and traumatic reactions}

\begin{abstract}
Serious stressful conditions in children, like neglect, emotional or sexual abuse within the family can result in traumas with symptoms that are currently not included in post-traumatic stress disorder (PTSD). The consequences of this complex trauma affect psychological development, and their characteristics are actually diagnosed in the category of non-specific extreme posttraumatic stress disorder (DESNOS, Pelcovitz et al., 1992), alterations of which include: a) emotional regulation and impulse control; b) memory and attention; c) self-perception; d) interpersonal relationships; e) somatization, and f) system of meanings. These symptoms are included in the proposal of a new diagnostic category: extreme or complex posttraumatic stress disorder (CPTSD). The specific characteristics of this disorder in minors that present complex trauma are being investigated (Cook et al., 2005). Data are presented on the symptomatology of complex trauma in maltreated minors.
\end{abstract}

Keywords: posttraumatic reactions, PTSD, complex trauma, DESNOS, childhood abuse.

\section{ESTRÉS Y REACCIONES TRAUMÁTICAS}

Si en cualquier época del desarrollo de la persona la presencia de acontecimientos estresantes o situaciones vitales adversas es importante en relación a la salud física y psicológica, durante la infancia su impacto puede ser dramáticamente significativo, ya que no va a afectar a un ser humano biológica, psicológica y so-

Recibido 14 febrero 2008; aceptado 31 julio 2008

Correspondencia: Concepción López-Soler, Universidad de Murcia, Facultad de Psicología, Dpto. Personalidad, Campus de Espinardo, Edif. Luis Vives, Apartado 4021. Correo-e: clopezs@um.es cialmente maduro, sino a un ser humano en una fase de desarrollo que requiere ciertas condiciones externas de estabilidad y protección. Por ello cuando un menor sufre unas condiciones de crianza inadecuadas o claramente negativas, los efectos en su desarrollo como persona pueden ser muy graves, ya que influyen en un gran número de variables psicológicas, la maduración del sistema nervioso y neuroendocrino (van der Kolk, 2003), y generando graves problemas de personalidad y de adaptación.

Los sucesos vitales experimentados pueden ser extraordinarios y extremadamente traumáticos, sucesos menores de baja intensidad de estrés, pero cotidianos, y todas las combinacio- 
nes entre estos extremos (Sandín, 2008; Talarn, Navarro, Russell y Rigat, 2006). En ambos casos, además de las unidades de cambio vital que se asocien al estímulo o situación estresante, para comprender su impacto en una persona concreta es necesario tener en cuenta las variables psicológicas, básicamente de tipo cognitivo (Sandín, Rodero, Santed y García-Campayo, 2006), que median entre los estímulos y las respuestas de estrés, tal como propuso Lazarus en su perspectiva interaccional (Lazarus y Folkman, 1984). Así la evaluación primaria del acontecimiento determina su valor de amenaza, daño/pérdida, desafío o beneficio, mientras que la secundaria valora la controlabilidad del estímulo y los recursos disponibles para afrontar la situación. Estos procesos cognitivos de gran importancia en la resolución del conflicto generado entre demandas del ambiente y recursos personales, durante la infancia están bastante limitadas, ya que las características del desarrollo cognitivo y afectivo en cada fase evolutiva impone claros límites, siendo fundamental la guía y protección de los adultos responsables del menor, porque en gran medida el significado de los sucesos y las reacciones infantiles, van a depender de los significados que sus cuidadores atribuyan al suceso y de sus reacciones.

Por otro lado, son importantes algunas características de los acontecimientos vitales tales como su duración, frecuencia, cantidad, intensidad y predictibilidad, así como su origen: naturales (inundaciones, terremotos, etc.) o humanos (terrorismo, maltrato, violación, etc.). Esta última característica es muy relevante como predictor de las reacciones al estrés, ya que el hecho de ser otra persona quien determine el daño y que esa persona mantenga un vínculo afectivo significativo, agrava la reacción y la hace más compleja. (Para una presentación actualizada sobre estas cuestiones, véase Sandín, 2008).

Las reacciones de las personas ante situaciones vitales adversas o acontecimientos naturales catastróficos son muy variadas y dependen de distintas características de fuentes diferentes: condiciones específicas del suceso, momento particular en el que se produce, ambiente cultural y social, fase evolutiva, etc. (Labrador y Alonso, 2007). En general se consideran normales las siguientes reacciones ante un evento estresante grave: tristeza, ansiedad, enfado, comportamiento alterado y otras dificultades menores, que perturban durante un breve periodo de tiempo. Estas reacciones transitorias son muy frecuentes en los menores y su superación, estabilidad o agravamiento depende en gran medida de la actitud de los padres o cuidadores para hacer que los/as hijos/as sientan protección y seguridad (Dyregrow y Yule, 2006).

Sin embargo a veces las dificultades son más intensas y duran más tiempo, provocando serios problemas en el funcionamiento personal y en la adaptación psicosocial. En estos casos, es además frecuente que esas reacciones conformen un conjunto de síntomas que permiten el diagnóstico de un trastorno psicopatológico específico como el trastorno adaptativo, el trastorno por estrés postraumático o la reacción al estrés aguda.

\section{EL TRASTORNO POR ESTRÉS POSTRAUMÁTICO EN LA INFANCIA Y ADOLESCENCIA}

El trastorno por estrés postraumático (TEPT), no había sido descrito en las clasificaciones oficiales hasta la década los 80, en el Diagnostic and Statistical Manual of Mental Disorders (DSM-III, American Psychiatric Association, APA, 1980), y fue recogido en gran medida por la presión ejercida por los veteranos de Vietnam. Anteriormente se aceptaba la categoría «gran reacción al estrés» en el DSM-I (APA, 1952), y «trastorno situacional transitorio» en el DSM-II (APA, 1968). La Organización Mundial de la Salud (OMS), no lo incluye hasta la novena versión en 1977, de la Clasificación Internacional de las Enfermedades (CIE), en la categoría «reacción aguda ante gran tensión». $\mathrm{La}$ última clasificación de la OMS, la CIE-10 (1992), propone una categoría de los trastornos provocados por estrés y trauma, en los cuales se incluye el TEPT agudo y crónico, los trastornos de adaptación y los cambios duraderos de personalidad posteriores a una situación catastrófica (F62.0). La consideración de que se produzca un impacto tan fuerte que provoque cam- 
bios en la personalidad, es de gran importancia en el desarrollo infantil, ya que situaciones muy adversas, cercanas y cotidianas, no sólo determinan síntomas y alteraciones psicológicas, sino que comprometen el desarrollo completo de la personalidad.

En la infancia y en temas de salud, se han descrito tempranamente reacciones traumáticas en menores intervenidos quirúrgicamente (Levy, 1945), y más actualmente en menores con cáncer (Pot-Mess, 1989; Stuber, Meeske, Gonzalez, Houskamp y Pynoos, 1995). Muy tempranamente se observó que aquellos menores que viven en condiciones amenazantes, la falta de calma y control por parte de los adultos que le rodean (Solomon, 1942; Mercier y Despert, 1943; López-Soler, 2004), hace que se vean más afectados que cuando los adultos reaccionan equilibradamente. Se destaca en estos relatos la importancia en la infancia del impacto de acontecimientos vitales cotidianos frente a un acontecimiento excepcional. En los años 70, se informó de las reacciones de menores ante situaciones gravemente estresantes, por ejemplo ante el hundimiento de un colegio (Lacey, 1972), o ante inundaciones (Newman, 1976). Pero fueron las investigaciones de Leonore Terr $(1979,1983,1985)$, sobre las reacciones de los menores que fueron secuestrados en un autobús escolar en 1976 en Chowchilla (California), el inicio de un enfoque específico y fructífero sobre el TEPT en la infancia. Terr informó que el 100\% de los menores secuestrados presentaban síntomas del trastorno, lo que permitió comprender que cuando la situación estresante es lo suficientemente intensa y duradera, y el menor lo experimenta de forma directa, aparece esta sintomatología descrita por la APA, en todos los casos, aunque sus características y evolución no es idéntica.

En general, los datos sobre prevalencia del TEPT en la infancia han sido muy variables, y se estima que hay más estudios en adolescentes mayores y adultos. Según Kessler, Sonnega, Bromet, Hughes y Nelson (1995), aproximadamente el $10 \%$ de jóvenes y adultos estadounidenses presentan sintomatología TEPT. Giaconia et al., (1995) informan que en adolescentes la prevalencia es del 6\% aproximadamente. En jóvenes daneses la prevalencia encontrada es del 9\% (Elklit, 2002). En contraste, el British Nacional Survey of Mental Health, en un estudio realizado sobre 10.000 menores informó que solo el $0,4 \%$ de niños/as de edades comprendidas entre los 11 y 15 años, fueron diagnosticados de TEPT, siendo el doble de frecuente en chicas que en chicos (Meltzer, Gatward, Goodman y Ford 2000). Por debajo de los 10 años se ha estudiado poco y no se dispone de buenos instrumentos de medida para ello (Dyregrov et al., 2006). Más fácil es encontrar estudios en la infancia sobre trauma, cuando se produce un desastre natural, así La Greca y Prinstein (2002), estimaron que en esas situaciones, entre el 30 y el 50\% de jóvenes desarrollan TEPT, y entre un 5 y un 10\% de menores. Najarian, Goenjian, Pelcovttz, Mandel, y Najarian (1996), informaron que el 90\% de los jóvenes presentaron síntomas severos del TEPT posterior a la invasión Armenia. Pero no siempre la respuesta del ser humano ante eventos graves es el TEPT. Más frecuentemente encontramos problemas de ansiedad, depresión, agresividad y consumo de sustancias en los menores (Reijneveld, Crone, Verlhust y Verloove-Vanhorick, 2003). En este sentido, Copeland, Kee1ler, Angold y Costello et al., (2007), informan que un ambiente adverso, múltiples situaciones de riesgo, problemas parentales y reacciones depresivas previas, son predictores del TEPT. Estos autores informan de presencia de sintomatología TEPT en un $13,4 \%$, y un $0,5 \%$ de TEPT en menores que han tenido experiencias estresantes, y consideran que las reacciones postraumáticas en infancia y adolescencia pueden expresarse en diferentes formas de psicopatología, presentando una fuerte asociación con alteraciones depresivas y ansiosas.

Lansford, Dodge, Bates, Croziery y Kaplow (2002), realizaron un estudio prospectivo a lo largo de 12 años, observando que el 74\% de los adolescentes que habían sufrido maltrato físico presentaron algún problema de conducta frente al $43 \%$ de los chicos que no habían sufrido maltrato físico. Además presentaron más de tres problemas de conducta el $21 \%$ de los menores con maltrato frente al 7\% del grupo que no recibió abuso físico. Las chicas que sufrieron abuso físico o sexual podían presentar en mayor medida problema de conducta $(\mathrm{OR}=7,1)$, depre- 
sión $(\mathrm{OR}=7,2)$, ansiedad $(\mathrm{OR}=9,3)$ o estrés postraumático $(\mathrm{OR}=9,8)$. En nuestro medio, se ha comprobado retraso en el desarrollo psicológico de menores de 5 años que sufrieron abuso (Osuna, Cabrera y Morales, 2000), y se estima que el riesgo de tentativa de suicidio en adolescentes maltratados es cinco veces más alto, comparado con aquellos que no presentaron maltrato en la infancia.

Los síntomas que se consideran característicos del TEPT son: la re-experimentación del acontecimiento (p.ej., flashbacks, pesadillas, etc.), la evitación (de recuerdos y situaciones relacionadas con el acontecimiento traumático), el embotamiento afectivo, y un incremento en la activación (hiperactivación fisiológica, problemas de sueño, etc.). Sin embargo en los menores (niños/as y adolescentes), el efecto del trauma y su valoración como TEPT puede adoptar la forma de un comportamiento desestructurado o agitado y presentarse con síntomas físicos o como sueños terroríficos o pesadillas, resultado de la expresión del síntoma «vivencia de horror»; conductas repetitivas, juegos simbólicos de los sucesos traumáticos, como expresión de la reexperimentación del suceso (Dyregrow y Yule, 2006), y disminución de intereses y retraimiento afectivo, inquietud, falta de atención y problemas de sueño.

Mientras que algunas formas y tipos de maltrato y abuso, pueden provocar en los menores alteraciones psicológicas compatibles con el modelo de TEPT, cuando los abusos son muy graves (maltrato físico/emocional extremo, abuso sexual), por intensos y cotidianos (por ser el/la abusador/a una persona muy cercana como madre, padre, otros familiares), pueden afectar de forma más dramática, comprometiendo todo el desarrollo de su personalidad, ya que conforman un ambiente del que no es posible escapar, y se establece como el mundo de referencia, sin otro tipo de significados. No resulta difícil comprender que en estos menores se desarrolle un síndrome de Estocolmo de adaptación primaria, ya que no han tenido otra posibilidad de estructurar su mapa de conocimiento y experiencias cognitivas y afectivas.

Según Finkelhor (1988), el maltrato grave y continuo (y en su caso, sexual), ocasiona perdida de confianza hacia otras personas, sentimiento de indefensión y desamparo, sexualización traumática, al incorporar aspectos sexuales en las relaciones interpersonales precozmente, que suele comportar dificultades para establecer relaciones íntimas normales y estigmatización personal, por cuanto se da sensación de vergüenza y culpa, junto a la consideración de haber sido responsable de los hechos.

Otra consecuencia a largo plazo del maltrato es la violencia transgeneracional, un niño maltratado tiene alto riesgo de ser perpetrador de maltrato en la etapa adulta a su pareja o a sus hijos, mientras que en las niñas está menos clara su evolución. Un meta-análisis publicado en Lancet (Ertem, Leventhal y Dobbs, 2000), encontró evidencia de que existe continuidad intergeneracional del abuso infantil, el riesgo relativo de transmisión del abuso de una generación a otra es de 12,6 (95\% IC: 1,82-87,2); por otro lado entre el 25 y el $50 \%$ de los niños/as sometidos a maltrato grave se encontraban asintomáticos, no presentando alteraciones emocionales o desordenes psiquiátricos en el momento de la valoración, lo que aporta una importante base para considerar la relevancia de las variables positivas de resiliencia, fortaleza y crecimiento en reacción al trauma $(\mathrm{Be}-$ coña, 2006; Harvey, 2007; Oliva, Jiménez, Parra y Sánchez-Queija, 2008). Sin embargo, la afectación negativa del maltrato es evidente: en los estudios que relacionan violencia doméstica con salud mental, se encuentra una alta relación entre la experiencia de malos tratos y problemas psicológicos, apareciendo trastorno de estrés postraumático, depresión, ansiedad, problemas de sueño, trastornos de somatización, entre otros (Campbell, 2002; Koss, 1990; Gomel, 1998; Mullen, Roman-Clarkson, Walton y Herbison, 1988; Ratner, 1993).

Después de la introducción de la categoría TEPT descrita por la APA con la tríada de síntomas y una fase de alternancia entre la reexperimentación y el embotamiento descrito por Horowitz (1976), se tomó en consideración este diagnóstico en personas con trauma por violación, violencia domestica, abuso infantil, incesto, etc. De esta forma síndromes postraumáticos descritos en diversos estudios, tales como: síndrome de trauma por violación (Burguess y Holmstrom, 1974), síndrome de violen- 
cia doméstica a mujeres (Walter, 1984), trauma de abuso sexual a menores (Briere, 1984,1987; Finkelhor 1985) y trauma por incesto (Courtois 1979a,b; Herman y Hirshman 1977), quedaban incluidos en el nuevo diagnóstico (APA 1980). Sin embargo muchos de estos autores no consideraron que la categoría diagnóstica de TEPT fuese totalmente adecuada para describir las reacciones nucleares experimentadas por víctimas de abuso o violación sistemática y mantenida entre seres humanos, es decir, para definir las consecuencias de los traumas ocurridos de forma reiterada e intensa, $y / o$ perpetrados por personas con las cuales la víctima mantenía un vínculo afectivo (Finkelhor, 1984; Herman, 1992a,b).

El abuso o maltrato íntimo, doméstico o familiar, se da durante largos periodos de tiempo, en los cuales los menores quedan atrapados y condicionados por un gran número y variedad de circunstancias estresantes. Debido a que la víctima es psicológica y físicamente inmadura, su desarrollo queda seriamente comprometido por el abuso repetido y la respuesta inadecuada por parte de algunos miembros de la familia o de otros cuidadores, por ejemplo cuando la madre niega o acepta la relación incestuosa del padre con una hija. A este fenómeno, en el que el/la menor se encuentra inmerso/a en un ambiente en el cual el trauma ocurre repetida y acumulativamente se denomina «trauma complejo» (Courtois 2004) y hace referencia a algunas formas de trauma muy complicadas y de consecuencias psicológicamente generalizadas (Herman 1992a, 1992b). En estos casos el trauma psicológico grave, determinaría un diagnóstico de reacción postraumática compleja, y se desarrolla cuando las condiciones vitales desbordan las posibilidades de entender y asimilar cognitiva y emocionalmente la experiencia, alterando la percepción de si mismo, de la afectividad y del mundo (Finkelhor y Browne, 1985).

Así, en la infancia, el abuso sexual y otras formas de abuso y maltrato, los conflictos armados, el haber sido prisionero de guerra, el secuestro, las violaciones, los desplazamientos por limpieza étnica, el ser refugiado, el tráfico humano y la prostitución, pueden provocar enfermedades físicas y trastornos psicopatológicos muy graves y de difícil diagnóstico y tratamiento.
En Estados Unidos, un estudio muestra que los efectos psicológicos de la violación son comparables con los efectos de las torturas o el rapto (Breslau et al., 1998). En un estudio sobre menores que habían sido victimas de acoso y abuso sexual (17 niños y 28 niñas), se encontró que el $100 \%$ presentó indicadores emocionales relativos a ansiedad, sentimiento de inadecuación y agresión; el $62 \%$ baja autoestima; el $62 \%$ interés sexual, el 50\% miedo; el 46\% dependencia; el 36\% sintomatología depresiva; el 33\% confusión de la identidad sexual; el $27 \%$ culpabilidad, y el 15\% miedo al ataque sexual (Petrzelová, 2005).

En nuestro país algunos estudios aportan datos en este sentido y señalan que el desarrollo del TEPT como consecuencia de cualquier abuso lo experimenta el $25 \%$ de todas las víctimas, pero que este porcentaje asciende hasta el 50$60 \%$ en el caso de mujeres y niñas agredidas sexualmente (Corral, Echeburúa, Sarasua y Zubizarreta, 1992) y hasta el 51-55\% en el caso de víctimas de maltrato doméstico (Amor, Echeburúa, Corral, Zubizarreta y Sarasua, 2002; Zubizarreta et al., 1994). Así mismo señalan que cuando las condiciones estresantes son muy intensas y son causadas por un ser humano, el cuadro clínico resultante es más grave y duradero, ya que aumenta la percepción de incontrolabilidad (Echeburúa y Corral, 1998).

A pesar de que se considera bastante común en menores la vivencia de experiencias traumáticas (Finkelhor y Dziuba-Leatherman, 1994), es difícil establecer la prevalencia del impacto de los eventos estresantes en general y del TEPT en particular en la infancia, debido a que las medidas de autoinforme elaboradas hasta el momento son para adultos, y se consideran poco adecuadas para realizar una correcta evaluación en los menores (Foa, Jonson, Feeny y Treadwell, 2001). Se han publicado una gran cantidad de investigaciones sobre las consecuencias de los malos tratos intra-familiares, escolares y sociales en niños y niñas, y se han empleado un gran número de escalas, inventarios y cuestionarios para su evaluación. Durante los últimos 10 a 15 años, los investigadores interesados en traumas en la infancia y la adolescencia, han respondido con un gran número de instrumentos de evaluación (Strand, 
Sarmiento y Pasquale, 2005). Sin embargo se hace difícil elegir entre los diferentes instrumentos ya que cada uno de ellos atiende un aspecto específico del problema.

\section{INADECUACIÓN DEL DIAGNÓSTICO TEPT EN REACCIONES POSTRAUMÁTICAS GRAVES \\ EN LA INFANCIA Y ADOLESCENCIA: PROPUESTA DE TRASTORNO POSTRAUMÁTICO COMPLEJO O EXTREMO}

Herman (1992a, 1992b), a partir de diversos estudios factoriales sobre traumas por abuso en la infancia, determinó que las principales consecuencias psicológicas no se recogían en el TEPT de la APA, y que las características principales del trauma eran: depresión, ansiedad, odio hacia sí mismo, disociación, abuso de sustancias, conductas autolesivas y comportamientos de riesgo, revictimización, problemas interpersonales y en las relaciones íntimas (incluidos familiares), preocupaciones somáticas y desesperación o desesperanza. En mujeres que han sufrido violencia familiar, se detecta un perfil psicopatológico caracterizado por trastorno por estrés postraumático, síntomas de ansiedad y depresión, y baja autoestima (Amor, Echeburúa, Corral, Sarasua y Zubizarreta, 2001). Sin embargo, estas características fueron entendidas como condiciones de comorbilidad más que como elementos esenciales de la complicada y compleja adaptación postraumática. Pero como estas condiciones son muy comunes en victimas de maltrato y son precisamente las más difíciles de tratar en terapia, se ha propuesto que este conjunto de síntomas en realidad conforman un trastorno por estrés postraumático complejo (CPTSD), que actualmente se diagnostica en la categoría de trastorno por estrés extremo no especificado (disorders of extreme stress not otherwise specified, DESNOS) (Pelcovitz et al., 1997), y que está siendo objeto de diversos ensayos de campo propiciados por la APA, con objeto de validar el síndrome.

Los efectos de la violencia pueden ser diferentes en función del grado de amenaza que suponga y de lo habitual que sea. Un maltrato de nivel medio y habitual podría determinar síntomas depresivos y de ansiedad, incluido el TEPT. Cuando las amenazas aumentan en intensidad y frecuencia, también se incrementa la probabilidad de que la víctima incorpore el sistema de creencias del agresor de modo defensivo (síndrome de Estocolmo). No aceptar parte de las creencias del agresor/a desestabiliza más. Si las experiencias son extremas y reiteradas la víctima se desconectaría de sus sentimientos y mostraría «entumecimiento psíquico», síntomas disociativos, miedo y desconfianza pudiendo configurar síntomas y patología de tipo psicótico. Es más probable que se experimentaran síntomas de TEPTC si la victimización se ha dado en una etapa temprana, ha sido prolongada en el tiempo y ha sido de naturaleza interpersonal.

Por otra parte, las relaciones entre la sintomatología TEPT y TEPTC son variadas: puede existir alta comorbilidad entre el trastorno de estrés complejo o extremo y el TEPT (Roth, Newman, Pelcovitz, van der Kolk y Mandel, 1997), y también puede desarrollarse el TEPTC sin sintomatología TEPT (Ford, 1999). Las alteraciones psicopatológicas que actualmente pueden diagnosticarse en el Trastorno por Estrés Postraumático No Especificado (DESNOS), y que formaran parte del nuevo diagnóstico de Trastorno por Estrés Postraumático Complejo, se han establecido en base a las propuestas independientes de Herman y van der Kolk (1987), en Boston, y de Spitzer, Kaplan y Pelcovitz (1989), en Nueva York. Aunque el procedimiento fue diferente, en ambos casos se basaron en el análisis de los síntomas que sistemáticamente mostraban personas que habían sufrido exposición prolongada a situaciones potencialmente traumáticas y habían desarrollado reacciones postraumáticas graves.

Las alteraciones centrales del trastorno por estrés postraumático complejo son las siguientes (Herman, 1992a,b):

(1) Alteraciones en la regulación de los impulsos afectivos. Incluye dificultad para modular la rabia y las conductas autodestructivas. Este síntoma indica déficit en la regulación emocional y el autocontrol e incluye las adicciones y comportamiento autolesivo, que son, paradójicamente, a menudo, usadas como salvavidas. 
(2) Alteraciones en la atención y la consciencia, tales como embotamiento, lentitud procesamiento, dificultades atención y concentración, amnesias, episodios disociativos y despersonalización. Se considera que la disociación tiende a ser un mecanismo de defensa ante el abuso interpersonal prolongado y severo ocurrido durante la infancia.

(3) Alteraciones en la autopercepción, así como un sentido crónico de culpabilidad y de responsabilidad personal, que cursa con sentimientos de intensa vergüenza. Los individuos abusados crónicamente, a menudo interiorizan el abuso como una forma de autovaloración (Courtois, 1979a, 1979b; Pearlman, 2001).

(4) Alteraciones en la percepción del maltratador. Incluye aceptación, dependencia e incorporación de su sistema de creencias. Estas características organizan las relaciones complejas, el sistema de creencias y posibilitan los abusos premeditados que continúan de forma repetitiva a manos de los cuidadores primarios, en caso de menores.

(5) Alteraciones en las relaciones con los otros. Dificultades para confiar e intimar, desarrollo de fuerte sensación de vulnerabilidad y peligro cuando inician nuevas relaciones afectivas en la medida que se hacen más intensas e intimas. Perciben que las personas con las que se relacionan afectivamente pueden utilizarlos y dañarles sin respeto y consideración de sus propias necesidades.

(6) Somatización y/o problemas médicos. Estas reacciones somáticas y condiciones médicas pueden explicar directamente el tipo de abusos sufridos y algún daño físico, o bien puede ser más difusa y aparecer como somatizaciones.

(7) Alteraciones en el sistema de significados. Los individuos abusados crónicamente, a menudo sienten una gran desesperanza acerca del mundo y del futuro. Así mismo creen que no encontraran a nadie que les entienda o entienda su sufrimiento. Mantienen un gran conflicto interno, con niveles de angustia altos, e intentan encontrar a alguien que les ayude a recuperarse de su angustia psíquica.

Podríamos considerar que algunas de estas características suponen mecanismos adaptati- vos que estructuran el significado del mundo y de las relaciones en un proceso de aprendizaje patológico, que está siendo legitimado por el resto de personas consentidoras. De esta mane$\mathrm{ra}$, los síntomas disociativos proporcionan una respuesta protectora natural a la arrolladora experiencia traumática, que surgen como una respuesta automática al estrés, en este sentido aparecen como reacciones disociativas primarias el olvido, la fragmentación y el embotamiento emocional (Kisiel y Lyons, 2001), precediendo o acompañando a la despersonalización, desrealización y amnesia. Si es complicada la evaluación del TEPT en la infancia, más difícil resulta encontrar instrumentos de evaluación adecuados y psicométricamente fiables para analizar los diferentes síntomas del trastorno por estrés postraumático complejo (TEPTC).

Hasta el momento los síntomas centrales del TEPTC propuesto, se consideran de diferentes maneras. Para algunos autores son síntomas asociados y comorbilidad del TEPT (DSM-IV; Copeland, Keeler, Angold y Costello, 2007); para otros representan síntomas de diferentes tipologías de las reacciones postraumáticas (Portnova, 2007); reconociendo que el diagnóstico incluye todos los síntomas conjuntamente, y aceptando la posibilidad de que se establezcan cluster específicos internalizantes (ineficacia, vergüenza, desesperación, desesperanza, retraimiento y quejas somáticas), y/o externalizantes (autodestrucción, agresividad, conducta impulsiva, hostilidad) (Miller y Ressik, 2007; Moran, 2007).

El diagnóstico de TEPTC, a pesar de no estar incluido en el DSM-IV, sigue encontrando apoyo en diversas investigaciones, por considerar que responde a la adaptación post-traumática en abuso severo y trauma afectivo en la infancia (Briere, 1984; Cook et al., 2005; Copeland et al., 2007; Herman, Perry y Van der Kolk, 1989; Kroll, 1993; van der Kolk, Perry y Herman, 1991; van der Kolk, Roth, Pelkovitz, Sundey y Spinazzola, 2005; Zanarini, 1997). Algunos autores consideran que las tendencias suicidas, las conductas de riesgo, la revictimización, la labilidad emocional, la impulsividad y la inestabilidad afectiva, generada como respuesta al estrés grave, coinciden con las características del trastorno límite de la personali- 
dad, por ello se ha establecido un gran y adecuado debate sobre las relaciones entre estos trastornos del eje I y II. Algunos autores consideran que personas que han recibido el diagnostico TEPT crónico (una de las denominaciones de las reacciones traumáticas graves, complejas y/o extremas), presentan a partir de la adolescencia trastorno límite de la personalidad (Hodges, 2003). Y puesto que la afectación de la personalidad es muy importante en la consideración del TEPTC, algunos investigadores han realizado estudios sobre la relación existente entre experiencias traumáticas y trastornos clínicos y de personalidad. Allen, Hunton y Evans (1999), aplicaron a mujeres con trastornos postraumáticos graves, el Inventario Clínico Multiaxial de Millón y hallaron cinco cluster que denominaron alienación, agresividad, retraimiento, viztimización y adaptación. Los dos primeros, de tipo externalizante, se caracterizan por altas puntuaciones en las escalas de agresividad, trastornos de la personalidad antisocial y límite, consumo de alcohol y drogas, y manía. Los cluster retraimiento y victimización, de tipo internalizante, agrupan las escalas trastornos de la personalidad depresivo, evitativo y esquizoide, con puntuaciones altas, y con bajas puntuaciones en trastornos de la personalidad histriónico y narcisista. El quinto cluster, adaptación, se caracteriza por bajas puntuaciones en trastornos de la personalidad y síndromes clínicos, caracterizándose por sintomatología postraumática simple. Estos hallazgos coinciden con los obtenidos en las investigaciones de Miller (Miller, Greif y Smith, 2003; Millar, Kaluepek, Dillon y Keane, 2004; Millar, y Ressik, 2007).

La consideración de diversos tipos en las reacciones postraumáticas, con diferente grado de afectación y curso es de gran importancia tanto a nivel diagnóstico como terapéutico. A nivel diagnóstico por que la ausencia de sintomatología TEPT y la presencia de síntomas subclínicos depresivos, ansioso, y conductas agresivas y hostiles, pueden estar indicando un trastorno más grave y complicado terapéuticamente que el TEPT. Por otro lado también es importante en el planteamiento terapéutico, ya que la exposición directa al trauma, tal como se recomienda en el tratamiento del TEPT, podría determinar retraumatizaciones (Chu, 1998; Courtois, 1999; 2004), en personas que presentan TEPTC, si previamente a la exposición, no es regulado y fortalecido antes el sistema afectivo, la estabilidad y la identidad personal.

En adultos las reacciones postraumáticas complejas ya han sido estudiadas, y se defiende el diagnóstico de trastorno por estrés extremo no especificado, DESNOS (disorders of extreme stress not otherwise specified), cuyos síntomas se describen en la Tabla 1.

Tabla 1. Síntomas del trastorno de estrés postraumático complejo
AFECTOS E IMPULSOS:
Regulación Afecto
Modulación Ira
Autodestructividad
Preocupación suicida
Dificultad modulación sexualidad
Conductas de riesgo

\section{ATENCIÓN Y CONSCIENCIA:}

Amnesia

Episodios disociativos transitorios

Despersonalización

\section{AUTOPERCEPCIÓN: \\ Ineficacia \\ Culpa y responsabilidad \\ Vergüenza \\ Incomprensión \\ Minimización personal}

RELACIONES INTERPERSONALES:

Inhabilidad para relaciones intimas

Revictimización

Victimizar otros

SOMATIZACIONES:

Sistema digestivo

Dolor crónico

Síntomas cardiopulmonares

Síntomas conversión

Sistema reproductor

SISTEMA DE SIGNIFICADOS:

Desesperanza

Infelicidad

Perdida sistema previo creencias 
Este diagnóstico ha sido validado por Zlotnick y Perlstein (1997), y Blaustein, Spinazzola, Simpson y van der Kolk (2000); a través de la entrevista SIDES: Structured Interview of Disorders of Extreme Stress. Esta entrevista consta de 45 items y ha sido elaborada por Pelkovitz et al. (1997). Spinazzola, Blaustein, Kisiel y van der Kolk (2001), han realizado otra validación a través del Self Report Inventory for Disorders of Extreme Stress (SIDES-SR), obteniendo una puntuación en el coeficiente alfa de 0,93 , para la escala total.

En infancia y adolescencia Cook et al. (2005) proponen siete grupos de síntomas para el diag- nóstico TEPTC, que sintetizan las alteraciones centrales cuando los menores expuestos a condiciones de riesgo graves y crónicas, presentan reacciones postraumáticas complejas. Las áreas afectadas y los problemas principales asociados al trauma son: alteraciones en las relaciones afectivas y apego, en las reacciones biológicas (físicas y psicosomáticas), en la regulación del afecto y la autorregulación, en la consciencia, con síntomas disociativos, en el control de la conducta, en la cognición y en el autoconcepto (Tabla 2).

Tabla 2. Áreas afectadas y alteraciones en menores expuestos a trauma complejo (adaptado de Cook et al., 2005)

\begin{tabular}{lll}
\hline I. APEGO & IV. DISOCIACIÓN & VI. COGNICIÓN \\
Problemas con límites & Estado consciencia alterado & Dificultades regulación atención \\
Desconfianza, suspicacia & Amnesia & y FE* \\
Aislamiento social & Despersonalización, desrealización & Lack of sustained curiosity \\
Dificultades Interpersonales & Dos o más estados de consciencia & Probabilidad procesamiento \\
Dif. estados emocionales otros & Fallos de memoria sucesos & información nueva \\
Dificultad toma perspectiva & & Probabilidad concentración tareas \\
II. & CONTROL & complejas \\
Probabilidad desarrollo & Pobre regulación impulsos & Probabilidad constancia objetivos \\
psicomotor & Conducta autodestructiva & Dificultades planificación/ anti- \\
Analgesia & Agresividad hacia otros & cipación \\
Probabilidad tono/balance, & Prob. patológicos auto-calma & Probabilidad comprensión \\
coordinación & Problemas sueño & responsabilidades \\
Somatizaciones & Sumisión excesiva & Dificultades aprendizaje \\
Problemas médicos aumentados & Conducta oposicionista & Probabilidad desarrollo lenguaje \\
& Dificultades comprender/aceptar & Probabilidad orientación tiem- \\
REGULACIÓN AFECTO & normas & po/espacio \\
Dificultades autorregulación & Representa trauma & \\
emocional & conductas/juegos & VII. AUTOCONCEPTO \\
Dificultades reconocer/expresar & & Continuidad/predictibilidad self \\
sentimientos & & alterado \\
Probabilidad reconocer estados & & Pobre sentido separación \\
internos & & Alteración imagen corporal \\
Dificultades comunicar deseos & & Baja autoestima \\
y necesidades & & Vergüenza y culpa
\end{tabular}

Nota. *Funciones ejecutivas

\section{VALORACIÓN CLÍNICA DE LOS DIAGNÓSTICOS TEPT Y TEPTC EN LA INFANCIA MALTRATADA}

En base a un convenio establecido entre la Consejería de Política Social, Mujer e Inmigración y la Universidad de Murcia (Proyecto de Evalua- ción Diagnóstica y Tratamiento Psicológicos en Menores Tutelados, PEDIMET), se atienden a menores que han sufrido maltrato intrafamiliar crónico, de tipo físico, emocional y social; que presentan reacciones psicológicas graves y que residen en centros o en acogimiento familiar (familia extensa o ajena). Son remitidos por alteraciones externalizantes principalmente, sin em- 
bargo la sintomatología característica en estos menores desde la perspectiva clínica se caracteriza por: dificultad en la regulación de los impulsos (agresividad y rabia), problemas afectivos $\mathrm{y}$ de ansiedad, dificultad para el reconocimiento del daño y expresión emocional, problemas en la atención, concentración y consciencia, dificultades en las relaciones interpersonales, problemas psicosomáticos y alteraciones en la percepción de los padres maltratadotes (justificaciones, idealizaciones, negación de la realidad...).

La experiencia en intervenciones psicológicas en menores que han sufrido experiencias traumáticas (malos tratos en la familia, abuso sexual, negligencia, abandono, rechazos en acogimiento en familia extensa o ajena), apoya más la propuesta del trauma y trastorno complejo, que la comorbilidad entre el TEPT y sintomatología diversa internalizante y externalizante. Sin embargo existen pocas escalas que valoren el trauma grave y continuo en la infancia y adolescencia, de ahí la importancia de continuar trabajando en el desarrollo de instrumentos de evaluación y medida del DESNOS o trauma complejo en la infancia. Parece necesario realizar una evaluación exhaustiva de las áreas asociadas al trauma para llevar a cabo un diagnóstico que nos permita realizar una intervención adecuada con los menores.

Por otra parte, aunque el TEPT, es uno de los más significativos desórdenes relacionados con el trauma, es frecuentemente pasado por alto en los ámbitos de salud mental y se tiende a considerar la sintomatología internalizante (tristeza, ansiedad, somatizaciones) y la externalizante (agresividad, ira, oposicionismo, inquietud, falta de atención, comportamientos destructivos hacía otros/si mismo/a), por separado. Es recomendable realizar el diagnóstico de estos diferentes síntomas teniendo en cuenta las características del trastorno por estrés postraumático complejo, ya que entonces algunos de estos signos que a veces no llegan a tener entidad diagnóstica por ser de nivel subclínico, se estructuran como una entidad clínica claramente configurada.

Por este mismo hecho, y para una aplicación efectiva de los tratamientos (Zimmerman y Mattia, 1999), resulta de suma importancia una evaluación estructurada de los eventos potencialmente traumáticos y de la psicopatología relacionada (Talarn, Navarro, Rossell y Rigat, 2006). Para realizar un diagnóstico psicológico fiable y evaluar la eficacia de los tratamientos, es imprescindible elaborar un protocolo de evaluación que detecte los síntomas descritos en el TEPTC, en niños y niñas maltratados. Como es posible que las reacciones postraumáticas configuren un TEPT, un TEPTC sin presencia de sintomatología TEPT (Ford, 1999); o en comorbilidad con dicho trastorno (Roth et al., 1997); es preciso incluir pruebas de evaluación psicológica que detecten signos de los dos trastornos. En todos los casos es conveniente explorar las variables personales de resistencia a la adversidad.

Mientras que los diagnósticos realizados en el proyecto PEDIMET, realizados sin tener presente la categoría diagnostica TEPTC, describen múltiples síntomas externalizantes en comorbilidad con sintomatología internalizante (Tabla 3), parece que está afectado todo el desarrollo psicológico, y que las reacciones postraumáticas de estos menores, se comprenden y se tratan mejor desde el diagnóstico de TEPTC.

Tabla 3. Características frecuentes en menores maltratados. Proyecto PEDIMET

Trastornos psicopatológicos:

Trastorno por estrés postraumático

Depresión

Ansiedad

Trastorno por déficit de atención con hiperactividad

Oposicionismo Desafiante

Otros

\begin{tabular}{ll}
\multicolumn{2}{c}{ Sintomatología: } \\
Problemas Atención & Inquietud \\
Desorientación & Ambivalencia emocional \\
Desolación & Aturdimiento \\
Crisis de Ira & Oposicionismo \\
Confusión & Tristeza \\
Labilidad emocional & Baja tolerancia frustración \\
Culpabilidad & \\
(creencia merecer maltrato) & Irritabilidad, agresividad \\
Angustia & Aislamiento/sumisión \\
Negación de la realidad & Temores \\
Autodesprecio & Desconfianza \\
Autolesiones & Problemas Aprendizaje \\
Baja adaptación personal & \\
y social & \\
Actitud seductora relaciones interpersonales (Sexualidad)
\end{tabular}


Con el fin de realizar una valoración inicial de los síntomas centrales del diagnóstico actual del TEPTC o DESNOS, en menores maltratados, se ha elaborado un listado de 14 síntomas, y han sido evaluados al inicio de los tratamientos 44 menores de edades comprendidas entre 6 y 15 años. Se ha considerado la presencia de cada síntoma cuando se ha puntuado 3 o más de tres en una escala de 0 a 5 . La puntuación del coeficiente alfa de Cronbach para este listado de síntomas es de 0,83 . Los resultados nos indican que la mayor parte de los indicadores están presentes en más de la mitad de este grupo de menores maltratados (ver Tabla 4).

Tabla 4. Listado indicadores DESNOS para la infancia y adolescencia maltratadas

\begin{tabular}{lc}
\hline Alteración regulación afectos & $94,2 \%$ \\
Alteración regulación impulsos & $67,7 \%$ \\
Alteraciones consciencia & $70,6 \%$ \\
Alteraciones autopercepción & $61,8 \%$ \\
Alteración percepción maltratadotes & $51,7 \%$ \\
Alteraciones relación iguales & $64,7 \%$ \\
Problemas relaciones acogedores7educadores & $73,5 \%$ \\
Depresión & $49,9 \%$ \\
Ansiedad & $67,5 \%$ \\
Autolesiones & $8,8 \%$ \\
Abuso de sustancias & $0 \%$ \\
Comportamientos autodestructivos/riesgo & $20,6 \%$ \\
Victimización & $17,6 \%$ \\
Problemas relaciones de intimidad & $41,2 \%$ \\
Desesperanza & $58,8 \%$ \\
\hline
\end{tabular}

Aunque solo se trata de una valoración inicial, el hecho de que se reconozcan variables que no pueden ser incluidas en el TEPT, aporta una buena base que apoya la importancia de disponer de categorías diagnósticas adecuadas para las reacciones postraumáticas graves y para estimular investigaciones sobre este tema. En la actualidad y con el objetivo de realizar una correcta evaluación y tratamiento psicológico a menores maltratados se han elaborado dos protocolos: uno para la evaluación de reacciones postraumáticas y otro para el tratamiento psicológico mediante realidad virtual, ambos para menores maltratados (Proyecto PEDIMET-EMMA, 2007).

Es difícil realizar una evaluación de las posibles variables psicológicas afectadas en menores que han sufrido maltrato grave y continuo, y por tanto, un posible trauma complejo (López-Soler et al., 2007a,b). En un intento de tener en cuenta las posibles áreas alteradas, así como las de resiliencia, que modulan el impacto del estrés, se han seleccionado una serie de pruebas psicológicas que permiten reconocer sintomatología TEPT y DESNOS (ver Tabla 5). Las medidas pre y postratamiento en estas escalas, así como los perfiles internalizantes y externalizantes, están siendo analizados. Una de las mejores maneras de ayudar a disminuir el sufrimiento y la desorientación de los menores maltratados, es conocer lo más exhaustivamente posible los diferentes componentes psicológicos del daño emocional que determina el trauma complejo. Como acertadamente señaló Carlson (1997), la última meta de los esfuerzos para comprender y evaluar los problemas psicológicos de las personas que desarrollan alteraciones psicopatológicas, es ofrecerles un tratamiento lo más efectivo y eficiente posible.

Tabla 5. Protocolo de evaluación psicológica equipo PEDIMET

\begin{tabular}{|c|c|c|c|c|c|}
\hline $\begin{array}{c}\text { Exploración } \\
\text { general }\end{array}$ & $\begin{array}{c}\text { Estrés } \\
\text { postraumático }\end{array}$ & $\begin{array}{l}\text { Ansiedad / } \\
\text { depresión }\end{array}$ & $\begin{array}{c}\text { Área } \\
\text { adaptación- } \\
\text { socialización }\end{array}$ & $\begin{array}{c}\text { Área afectivo- } \\
\text { emocional }\end{array}$ & $\begin{array}{l}\text { Variables } \\
\text { resiliencia }\end{array}$ \\
\hline $\begin{array}{l}\text { Entrevista } \\
\text { semiestructurada } \\
\text { de maltrato } \\
\text { (Martínez et al., } \\
\text { 2007) }\end{array}$ & $\begin{array}{l}\text { Escala Pediátrica } \\
\text { de Estrés } \\
\text { Emocional; } \\
\text { PEDS (Saylor } \\
\text { et al., 1999) }\end{array}$ & $\begin{array}{l}\text { Cuestionario } \\
\text { de Ansiedad } \\
\text { Estado-Rasgo } \\
\text { en niños; STAIC } \\
\text { (Spielberger, } \\
\text { 1990) }\end{array}$ & $\begin{array}{l}\text { Test } \\
\text { Autoevaluativo } \\
\text { Multifactorial } \\
\text { de Adaptación } \\
\text { Infantil; TAMAI } \\
\text { (Hernández, } \\
\text { 1999) }\end{array}$ & $\begin{array}{l}\text { Inventario de } \\
\text { Expresión de Ira- } \\
\text { Rasgo en Niños } \\
\text { y Adolescentes; } \\
\text { STAXI-NA } \\
\text { (Del Barrio, } \\
\text { Spielberger y } \\
\text { Aluja, 1998) }\end{array}$ & $\begin{array}{l}\text { Escala de } \\
\text { Resiliencia } \\
\text { (Wagnild y } \\
\text { Young, 1993) }\end{array}$ \\
\hline
\end{tabular}


Tabla 5. Protocolo evaluación psicológica equipo PEDIMET (Continuación)

\begin{tabular}{|c|c|c|c|c|c|}
\hline $\begin{array}{c}\text { Exploración } \\
\text { general }\end{array}$ & $\begin{array}{c}\text { Estrés } \\
\text { postraumático }\end{array}$ & $\begin{array}{l}\text { Ansiedad / } \\
\text { depresión }\end{array}$ & $\begin{array}{c}\text { Área } \\
\text { adaptación- } \\
\text { socialización }\end{array}$ & $\begin{array}{c}\text { Área afectivo- } \\
\text { emocional }\end{array}$ & $\begin{array}{c}\text { Variables } \\
\text { resiliencia }\end{array}$ \\
\hline $\begin{array}{l}\text { Escala de } \\
\text { maltrato } \\
\text { psicológico } \\
\text { (Walker, 1984; } \\
\text { adaptación } \\
\text { Garriga, et al., } \\
\text { 2007) }\end{array}$ & $\begin{array}{l}\text { CPSS } \\
\text { (The Child } \\
\text { PTSD Symptom } \\
\text { Scale; Foa et al., } \\
\text { 2001) }\end{array}$ & $\begin{array}{l}\text { CASI } \\
\text { (Índice de } \\
\text { Sensibilidad a la } \\
\text { Ansiedad Niños; } \\
\text { Sandín y Chorot, } \\
\text { 1997; Sandín et } \\
\text { al., 2002) }\end{array}$ & $\begin{array}{l}\text { BAS 1, 2, } 3 \\
\text { (Batería de } \\
\text { Socialización, } \\
\text { Silva y } \\
\text { Martorell, 2001) }\end{array}$ & $\begin{array}{l}\text { PANAS } \\
\text { (Escala de } \\
\text { Afecto Positivo y } \\
\text { Negativo; Sandín } \\
\text { et al., 1999) }\end{array}$ & $\begin{array}{l}\text { Escala de } \\
\text { autoeficacia } \\
\text { (Bandura, 1990) }\end{array}$ \\
\hline $\begin{array}{l}\text { STIA } \\
\text { (Escala de } \\
\text { Situaciones } \\
\text { Traumáticas } \\
\text { en la Infancia } \\
\text { y Adolescencia; } \\
\text { López-Soler, } \\
\text { 2006) }\end{array}$ & $\begin{array}{l}\text { CITES-R } \\
\text { (Children's } \\
\text { Impact Traumatic } \\
\text { Events Scale; } \\
\text { Wolfe et al., } \\
\text { 1991) }\end{array}$ & $\begin{array}{l}\text { CDI } \\
\text { (Cuestionario de } \\
\text { Depresión } \\
\text { Infantil; Kovacs, } \\
\text { 1994) }\end{array}$ & $\begin{array}{l}\text { Área } \\
\text { atención } \\
\text { y consciencia }\end{array}$ & $\begin{array}{l}\text { Diferencial } \\
\text { Semántico } \\
\text { Maltrato } \\
\text { García et al., } \\
\text { 2007) }\end{array}$ & $\begin{array}{l}\text { ACS } \\
\text { (Escala de } \\
\text { Afrontamiento } \\
\text { para } \\
\text { Adolescentes; } \\
\text { Rydenberg y } \\
\text { Lewys, 1996) }\end{array}$ \\
\hline $\begin{array}{l}\text { CBCL } \\
\text { (Child Behavior } \\
\text { Checklist; } \\
\text { Achenbach, } \\
\text { 1991) }\end{array}$ & $\begin{array}{l}\text { IES (Impact of } \\
\text { Event Scale, } \\
\text { Horowitz, Wilner } \\
\text { et Alvarez 1979) }\end{array}$ & $\begin{array}{l}\text { Síndromes } \\
\text { CBCL/YSR }\end{array}$ & $\begin{array}{l}\text { TAREA } \\
\text { DE STROOP } \\
\text { (Test de Colores } \\
\text { y Palabras; } \\
\text { Goleen, 2001) }\end{array}$ & & \\
\hline $\begin{array}{l}\text { YSR } \\
\text { (Youth Self } \\
\text { Report; } \\
\text { Achenbach, } \\
\text { 1991) }\end{array}$ & $\begin{array}{l}\text { SCARED-R } \\
\text { (Escala } \\
\text { abreviada de } \\
\text { cribado del } \\
\text { TEPT; Muris } \\
\text { 1997) }\end{array}$ & & $\begin{array}{l}\text { Subescalas } \\
\text { TAMAI }\end{array}$ & $\begin{array}{l}\text { Escala } \\
\text { de Experiencia } \\
\text { Disociativa } \\
\text { (Adapt. Berstein } \\
\text { y Putman, 1986) }\end{array}$ & $\begin{array}{l}1 .{ }^{\mathrm{a}} \text { parte } \\
\text { CBCL/YSR } \\
\text { BAS subescalas }\end{array}$ \\
\hline
\end{tabular}

\section{REFERENCIAS}

Achenbach, T., y Rescorla, A. (2001). Manual for the ASEBA School-Age Forms \& Profiles. Burlington, VT: University of Vermont.

Allen, J.G., Huntoon, J., y Evans, R.B. (1999). Complexities in complex posttraumatic stress disorder in inpatient women: Evidence from cluster analysis of MCMI-III personality disorder scales. Journal of Personality Assessment, 73, 449-471.

American Psychiatric Association (APA) (1952). Diagnostic and statistical manual of mental disorders. $\left(1^{\text {st }}\right.$ ed.). Washington, DC: Autor.

American Psychiatric Association (APA) (1968). Diagnostic and statistical manual of mental disorders. $\left(2^{\text {nd }}\right.$ ed.). Washington, DC: Autor.

American Psychiatric Association (APA) (1980). Diagnostic and statistical manual of mental disorder $\left(3^{\text {rd }}\right.$ ed.) (DSM-III). Washington, DC: Autor.
American Psychiatric Association (APA) (1994). Diagnostic and statistical manual of mental disorders $\left(4^{\text {th }} \mathrm{ed}\right.$.) (DSM-IV). Washington, DC: Autor.

Amor, P.J., Echeburúa, E., Corral, P., Sarasua, B., y Zubizarreta, I. (2001). Maltrato físico y maltrato psicológico en mujeres víctimas de violencia en el hogar: un estudio comparativo. Revista de Psicopatología y Psicología Clínica, 6, 167-178.

Amor, P.J., Echeburúa, E., Corral, P., Zubizarreta, I., y Sarasua, B. (2002). Repercusiones psicopatológicas de la violencia domestica en la mujer en función de las circunstancias del maltrato International Journal of Clinical and Health Psychology, 2, 227-246.

Bandura, A. (1990). Multidimensional Scales of perceived self-efficacy. Stanford, C.A.: Stanford University.

Becoña, E. (2006). Resiliencia: definición, características y utilidad del concepto. Revista de Psicopatología y Psicología Clínica, 11, 125-146. 
Blaustein, M.E., Spinazzola, J., Simpson, W., y van der Kolk, B.A. (2000). Psychological sequelae of early trauma: Comorbid diagnoser or diagnostic entity? Póster presentado en $16^{\text {th }}$ Annual Meeting of the International Society for Traumatic Stress Studies; San Antonio, TX.

Breslau, N., Kessler, R.C., Chilcoat, H.D., Schultz, L.R., Davis, G.C., y Andreski, P. (1998). Trauma and posttraumatic stress disorder in the community: The 1996 Detroit area survey of trauma. Archives of General Psychiatry, 55, 626-632.

Briere, J. (1984). The effects of childhood sexual abuse on later psychological functioning: Defining a post-sexual abuse syndrome. Póster presentado en Annual Convention of the American Psychological Association, Toronto, Ontario, Canadá.

Briere, J. (1987). Post-sexual abuse trauma: Data and implications for clinical practice. Journal of Interpersonal Violence, 2, 367-379.

Briere, J., y Spinazzola, J. (2005). Phenomenology and psychological assessment of complex posttraumatic states. Journal of Traumatic Stress, 18, 401-412.

Burgess, A.W., y Holmstrom, L.L. (1974). Rape trauma syndrome. American Journal of Psychiatry, 131, 981986.

Campbell, L. (2002). Health consequences of intimate partner violence. The Lancet, 359, 1331-1336.

Carlson, E.B. (1997). Trauma assessments: A clinician's guide. New York: Guilford.

Chu, J.A. (1998). Rebuilding shattered lives: The responsible treatment of complex post-traumatic and dissociative disorder. New York: Wiley.

Cohen, J.A., Mannarino, A.P., Greenbergt T, Padlo S, y Shipley C. (2002), Childhood traumatic grief: concepts and controversies. Trauma Violence Abuse, 3, 307-321.

Cook, A., Spinazzola, J., Ford, J., Lanktree, C., Blaustein, M., Cloitre, M., DeRosa, R., Hubbard, R., Kagan, R., Liautaud, J., Mallah, K., Olafson, E., y Van der Kolk, B. (2005). Complex trauma in children and adolescents. Psychiatric Annals, 35, 5.

Copeland, W.E., Keeler, G., Angold, A., y Costello, J. (2007). Traumatic events and posttraumatic stress in childhood. Archives of General Psychiatry, 64, 577-584.

Corral, P., Echeburúa, E., Sarasua, B., y Zubizarreta, I. (1992). Estrés post-traumático en excombatientes y víctimas de agresiones sexuales: nuevas perspectivas terapéuticas. Boletín de Psicología, 35, 7-24.

Courtois, C.A. (1979a). Characteristics of a volunteer sample of adult women who experienced incest in childhood and adolescence. Dissertation Abstracts International, 40A, Nov-Dec, 3194-A.

Courtois, C.A. (1979b). The incest experience and its aftermath. Victimology: An International Journal, 4, 337347.
Courtois, C.A. (1999). Recollections of sexual abuse: Treatment principles and guidelines. New York: Norton.

Courtois, C.A. (2004). Complex Trauma, Complex Reactions: Assessment and treatment. Psychotherapy: Theory, Research, Practice, Training, 41, 412-425.

Del Barrio, V., Spielberger, C.D., y Aluja, A. (2005). Inventario de expresión de ira estado-rasgo en niños y adolescentes, STAXI-NA. Madrid: TEA Ediciones.

Dyregrow, A., y Yule, W. (2006). A Review of PTSD in Children. Child and Adolescent Mental Health, 11, 176-184.

Echeburúa, E., Corral, P., Amor, P., Sarasua, B., y Zubizarreta, I. (1997) Repercusiones psicopatológicas de la violencia doméstica en la mujer: un estudio descriptivo. Revista de Psicopatología y psicología Clínica, 2, 7-19.

Echeburúa, E., y Corral, P. (1998). Manual de violencia familiar. Madrid: Siglo XXI.

Elklit, A. (2002). Victimization and PTSD in a Danish national youth probability sample. Journal of the American Academy of Child and Adolescent Psychiatry, 41, 174-181.

Ertem, I., Leventhal, J., y Dobbs, S. (2000). Intergenerational continuity of child physical abuse: how good is the evidence? The Lancet, 356, 814-819.

Finkelhor, D. (1984). Child sexual abuse: New theory and research. New York: Free Press.

Finkelhor, D. (1985). The traumatic impact of child sexual abuse: A conceptualization. Journal of Orthopsychiatry, 55, 530-541.

Finkelhor, D. (1988). The Adolescent Sexual Perpetrator: A new challenge in the field of sexual abuse. Treating the juvenile sexual abuse perpetrator: Proceedings of a national training conference. Bloomington, MN, April 27-30, 1986.

Finkelhor, D. (1988). The Trauma of Child Sexual Abuse: Two Models. En G. Wyatt, The lasting effects of child Sexual Abuse. Newbury Park, CA: Sage.

Finkelhor, D., y Browne, A. (1985). The traumatic impact of child sexual abuse: a conceptualization. Am J Orthopsychiatry, 55, 530-541.

Finkelhor, D., y Dziuba-Leatherman, S. (1994). Children as victims of violence: a national survey. Pediatrics, 94, 413-420.

Foa, E., Johnson, K.M., Feeny, N.C., y Treadwell, K.R. (2001). The Child PTSD Symptom Scale: A preliminary examination of its psychometric properties. Journal of Clinical Child Psychology, 30, 376-384.

Ford, J.D. (1999). Disorders of extreme stress following war-zone military trauma: Associated features of posttraumatic stress disorder or comorbid but distinct syndromes? Journal of Consulting and Clinical Psychology, 67, 3-12.

Frydenberg, E., y Lewis, R. (1996). Escalas de afrontamiento para adolescentes, ACS. Madrid: TEA Ediciones. 
García Montalvo, C., López-Soler, Martínez, A., y Garriga, A. (2006). Diferencial semántico maltrato infantil. Universidad de Murcia. Documento no publicado.

Garriga, A., López-Soler, C., Martínez, A., Fernández, V., Castro, M., y Puerto, J.C. (2007). Escala de maltrato psicológico; Adaptación de la Escala de Maltrato a Mujeres en L. Walker (1984). Universidad de Murcia. Documento no publicado.

Giaconia, R.M., Reinherz, H.Z., Silverman, A.B., Pakiz, B., Frost, A.K., y Cohen, E. (1995). Traumas and posttraumatic stress disorder in a community population of older adolescents. Journal of the American Academy of Child and Adolescent Psychiatry, 34, 1369-1380.

Golden, C.J. (2007). Test de colores y palabras, STROOP. Madrid: TEA Ediciones (5. ${ }^{\text {a }}$ edición).

Gomel, M.K. (1998). Domestic Violence-An Issue for Primary Health Care Professionals. En R. Jenkins y T.B. Ustun (Eds.), Preventing mental illness: Mental health promotion in primary care, (pp. 440-455). New York: Wiley.

Harvey, M.R. (2007). Towards and ecological understanding of resilience in trauma survivors: implications for theory, research and practice. En M.R. Harvey y P. Tummala-Narra (Eds.), Sources and expressions of resilience in trauma survivors: Ecological theory, multicultural practice. Journal of Aggression, Maltreatment, and Trauma, 14, 9-32.

Herman, J.L. (1992b). Trauma and recovery: The aftermath of violence-From domestic to political terror. New York: Basic Books.

Herman, J.L., Perry, J.C., y Van Der Kolk, B.A. (1989). Childhood trauma in borderline personality disorder. American Journal of Psychiatry, 146, 490-495.

Herman, J.L., y Hirschman, L. (1977). Father-daughter incest. Signs: Journal of Women in Culture and Society, 2, 735-756.

Herman, J.L., y van der Kolk, B.A. (1987). Traumatic antecedents of borderline personality disorder. En B. van der Kolk (Ed.), Psychological trauma (pp. 111-126). Washington, DC: American Psychiatric Press.

Hernan, J.L. (1992a). Complex PTSD: A syndrome in survivors of prolonged and repeated trauma. Journal of Traumatic Stress, 5, 377-391.

Hernández, P. (2004). Test Autoevaluativo multifactorial de adaptación infantil (TAMAI). Madrid: TEA Ediciones (5. ${ }^{\text {a }}$ edición, revisada).

Hodges, S. (2003). Borderline personality disorder and posttraumatic stress disorder. Time for integration? Journal of Counselling and Development, 81, 19-27.

Horowitz, M., Wilner, N., y Álvarez, W. (1979). Impact of Event Scale: A measure of subjective stress. Psychosomatic Medicine, 41, 209-218.

Horowitz, M.J. (1976). Stress-response syndromes. New York: Jason Aronson.
Jaycox, L. (2002). Violence exposure, posttraumatic stress disorder, and depressive symptoms among recent immigrants school children. Journal of the American Academy of Child Adolescent, 41, 1104-1110.

Kessler, R.C., Sonnega, A., Bromet, E., Hughes, M., y Nelson, C.B. (1995). Posttraumatic stress disorder in the National Comorbidity Survey. Archives of General Psychiatry, 52, 1048-1060.

Kisiel, C.L., y Lyons, J.S. (2001). Dissociation as a mediator of psychopathology among Sexually abused children and adolescents. American Journal of Psychiatry, 158, 1034-1039.

Koss, M.P. (1990). The women's mental health research agenda. Violence against women. American Psychology, 45, 374-380.

Kovacs, M. (2004). Inventario de Depresión Infantil (CDI). Madrid: TEA Ediciones.

Kroll, J. (1993). PTSD/borderlines in therapy: finding the balance. New York: Norton.

La Greca, A.M., y Prinstein, M.J. (2002). Hurricanes and earthquakes. En A.M. La Greca, W.K. Silverman, E.M. Vernberg y M.C. Roberts (Eds.), Helping children cope with disasters and terrorism. Washington: American Psychological Association.

Labrador, F., y Alonso, E. (2007). Evaluación de la eficacia a corto plazo de un programa de intervención para el trastorno de estrés postraumático en mujeres mexicanas víctimas de violencia doméstica. Revista de Psicopatología y Psicología Clínica, 12, 117-130.

Lacey, G. (1972). Observation on Aberfan. Journal of Psychosomatic Research, 16, 257-260.

Lansford, J.E., Dodge, K.E., Bates, J.E., Crozier, J., y Kaplow, J. (2002). A 12-year prospective study of the long term effect of early child physical maltreatment on psychological, behavioural and academic problem in adolescent. Archives of Pediatric and Adolescent Medicine, 156, 824-830.

Lazarus, M.S., y Folkman, S. (1984). Stress, appraisal and coping. New York: Springer.

Levy, D. (1945). Psychic Trauma of operation in children. American Journal of Diseases of Children, 69, 7-25.

López Soler C., García Montalvo, C., Pérez, A., Garriga, A., Alcántara, M.V., Fernández V., y Castro, M. (2007). Dificultades y ventajas de una evaluación psicológica amplia en menores traumatizados. 1er Congreso Internacional sobre Violencia, Abuso y Maltrato. Noviembre, Buenos Aires.

López Soler, C., Garriga, A., Puerto, J.C., Martínez, A., García Montalvo, C., Fernández, V., Castro y Alcántara, M. (2007). Alteraciones de la atención y la conciencia en infancia y adolescencia maltratada. 1er Congreso Internacional sobre Violencia, Abuso y Maltrato. Noviembre, Buenos Aires.

López-Soler, C. (2004). Intervención psicosocial y educativo con menores en alojamiento alternativo. En E. 
Pastor e I. Soler (Coord.), Intervención Psicosocial en mujeres maltratadas en alojamientos alternativos. Murcia: Consejería de Trabajo y Asuntos Sociales.

López-Soler, C. (2006). Escala de Situaciones Traumáticas Intrafamiliares. Universidad de Murcia. Documento no publicado.

López-Soler, C., Martínez, A., García Montalvo, C., Garriga, A., Alcántara, M.V., Fernández V., Castro, M., Prieto, M., y Puerto, J.C. (2007). Evaluación psicológica de menores maltratados: Estimación del daño emocional y de variables de resistencia personal frente a la adversidad. XLII Congreso de la Asociación Española de Psiquiatría Infantil y Juvenil, AEPIJ. Junio, Bilbao.

Martínez, A., López-Soler, C., García Montalvo, C, Garriga, A., Alcántara, M.V., y Prieto, M.(2006). Entrevista Semiestructurada de Maltrato Infantil y Adolescente. Universidad de Murcia. Documento no publicado.

Meltzer, H., Gatward, R., Goodman, R., y Ford, T (2000). Mental health of children and adolescent in Great Britain. London: The Stationery Office.

Mercier, M., y Despert, L. (1943). Effects of war on French children. Psychosocial Medicine, 5, 226-272.

Miller, M.W., Greif, J.L., y Smith, A.A. (2003). Multidimensional Personality Questionnaire profiles of veterans with traumatic combat exposure: Internalizing and externalizing subtypes. Psychological Assessment, 15 , 205-215.

Miller, M.W., Kaloupek, D.G., Dillon, A.L., y Keane, T.M. (2004). Externalizing and internalizing subtypes of combat related PTSD: A replication and extension using the PSY-5 Scales. Journal of Abnormal Psychology, 113, 636-645.

Miller, M.W., y Resick, P.A. (2007). Internalizing and externalizing subtypes in female sexual assault survivors: Implications for the understanding of complex PTSD. Behavior Therapy, 38, 58-71.

Moran, M. (2007). Developmental trauma merits DSM diagnosis, experts say. American Psychiatric Association. Psychiatric News, 42, 20.

Mullen, E.P., Roman-Clarkson, S.E., Walton, V.A., y Herbison, E.P.(1998). Impact of sexual and physical abuse on women mental health. Lancet, 1988, 842-845.

Muris, P. (1997). The screen for Child Anxiety Related Emotional Disorders (revised version). Maastricht: Maastricht University, Department of Psychology.

Najarian, L., Goenjian, A.K., Pelcovttz, D., Mandel, F., y Najarian, B. (1996). Relocation after a disaster: Posttraumatic stress disorder in Armenia after the earthquake. Journal of the American Academy of Child \& Adolescent Psychiatry, 35, 374-383.

Newman, C.J. (1976). Children of disaster: Clinical observations at Buffalo Creek. American Journal of Psychiatry, 133, 306-312.
Oliva, A., Jiménez, J.M., Parra, A., y Sánchez-Queija, I. (2008). Acontecimientos vitales estresantes, resiliencia y ajuste adolescente. Revista de Psicopatología y Psicología Clínica, 13, 53-62.

Osuna, M.J., Cabrera, J. H., y Morales, M. C. (2000). Estudio de las consecuencias del abandono físico en el desarrollo psicológico de niños en edad preescolar en España. Child Abuse and Neglect. The International Journal, 24, 911-924.

Pearlman, L.A. (2001). The treatment of persons with complex PTSD and other trauma-related disruptions of the self. En J.P. Wilson, M. Friedman, y J. Lindy (Eds.), Treating psychological trauma and PTSD. New York: Guilford Press.

Pelcovitz, D., Van Der Kolk, B.A., Roth, S., Mandel, F.S., Kaplan, S., y Resick, P.A. (1997). Development of a criteria set and a structured interview for disorders of extreme stress (SIDES). Journal of Traumatic Stress, 10, 3-17.

Portnova, A.A. (2007). Typology of post-traumatic stress disorder in children and adolescents. Neuroscience and Behavioral Physiology, 37, 7-11.

Pot-Mees, C. (1989). The psychological affects of bone marrow. Transplantation in children. Netherland: Eubron.

Pynoos, R.S., y Nader, K. (1993). Issues in the treatment of posttraumatic stress in children and adolescents. En J.P. Wilson y B. Raphael (Eds.), International handbook of traumatic stress syndromes. New York: Plenum Press.

Ratner, P.A. (1993). The incidence of wife abuse and mental health status in abused wives in Edmonton, Alberta. Canadian Journal of Public Health, 84, 246-249.

Reijneveld, S.A., Crone, M.R., Verhulst, F.C., y VerlooveVanhorick, P.S. (2003). The effect of a severe disaster of the mental health of adolescents: A controlled study. The Lancet, 362, 691-696.

Roth, S., Newman, E., Pelcovitz, D., Van der Kolk, B.A., y Mandel, F.S. (1997). Complex PTSD in victims exposed to sexual and physical abuse: Results from de DSM-IV field trial for posttraumatic stress disorder. Journal of Traumatic Stress, 10, 539-555.

Sandín, B. (Ed.) (2008). El Estrés psicosocial: Conceptos y consecuencias clínicas, 2. ${ }^{a}$ edición. Madrid: Klinik.

Sandín, B., Chorot, P., Lostao, L., Joiner, T.E., Santed, M.A., y Valiente, R.M. (1999). Escala PANAS de afecto positivo y negativo. Validación factorial y convergencia transcultural. Psicothema, 14, 333-339.

Sandín, B., Chorot, P., Santed, M.A., y Valiente, R.M. (2002). Análisis factorial confirmatorio del Índice de Sensibilidad a la Ansiedad para Niños. Psicothema, 14, 333-339.

Sandín, B., Rodero, B., Santed, M.A., y García-Campayo, J. (2006). Sucesos vitales estresantes y trastorno de pánico: Relación con la ocurrencia del trastorno de pá- 
nico, la gravedad clínica y la agorafobia. Revista de Psicopatología y Psicología Clínica, 11, 179-190.

Sandín, B., y Chorot, P. (1997). Versión Española del Índice de Sensibilidad a la Ansiedad (CASI). En B. Sandín, Ansiedad, Miedos y fobias en Niños y Adolescentes. Madrid: Dykinson.

Saylor, C.F., Cupit, C., Stokes, S., y Taylor, M. (1999). The Pediatric Emotional Distress Scale: a brief screening measure for young children exposed to traumatic events. Journal of Clinical Child Psychology, 28, 70-81.

Silva, F., y Martorell, M.C. (2001). Batería de Socialización. Autoevaluación (BAS-3). Madrid: TEA Ediciones (3. a edición).

Solomon J. (1942). Reaction of children to black-outs. American Journal Orthopsychiatry, 12, 361-362.

Spinazzola, J, Blaustein, M, Kisiel, C., y van der Kolk, B. (2001). Beyond PTSD: Further evidence for a complex adaptational response to traumatic life events. Póster presentado en American Psychiatric Association Annual Meeting, New Orleans.

Spitzer, R., Kaplan, S., y Pelcovitz, D. (1989). Victimization disorder: A needed addition to DSM-IV. Proceedings of the $142^{\text {nd }}$ Annual Meeting of the American Psychiatric Association Summary, 14, 234.

Strand, V.C., Sarmiento, T.L., y Pasquale, L.E. (2005). Assessment and screening tools for trauma in children and adolescent. A review Trauma, Violence and Abuse, 6, 55-78.

Stuber, M.L., Meeske, K., González, S., Houskamp, B.M., y Pynoos, R. (1995). Post-traumatic stress after childhood cancer I: the role of appraisal. Psychology Oncological, 3, 305-12.

Talarn, A., Navarro, N., Rossell, L., y Rigat, A. (2006). Propuesta de especificadotes diagnósticos vinculados al estrés y el trauma: Una aportación a la nosología psicopatológica. Revista de Psicopatología y Psicología Clínica, 11, 107-114.

Terr, L.C. (1979). Children of Chowchilla. A study of psychic terror. The Psychoanalytic Study of the Child, 34, 547-623.

Terr, L.C. (1983). Chowchilla revisited: The effect of psychic trauma four years after a school-bus kidnapping. American Journal of Psychiatry, 140, 1543-1550.
Terr, L.C. (1985). Children Traumatized en small groups. En S. Eth y R.S. Pynoos (Eds.), Post-traumatic stress disorders in children (pp. 47-70). Washington DC: American Psychiatric Press.

Van Der Kolk, B.A. (2003). The neurobiology of childhood trauma and abuse. Child and Adolescent Clinics of North America, 12, 293-317.

Van Der Kolk, B.A., Perry, J.C, y Herman, J.L. (1991). Childhood origins of self-destructive behaviour. American Journal of Psychiatry, 148, 1665-1671.

Van Der Kolk, B.A., Rothc, S., Pelcovitz, D., Sunday, S., y Spinazzola, J. (2005). Disorders of Extreme Stress: The Empirical Foundation of a Complex Adaptation to Trauma. Journal of Traumatic Stress, 18, 389-399.

Wagnild, G., y Young, H.M. (1993). Development and psychometrics evaluation of the Resilience Scale. Journal of Nursing Measurement 1, 165-177.

Walker, L. (1984). The battered woman syndrome. USA: Spring Publishing Company.

WHO (World Health Organization) (1992). The ICD-10. Classification of mental and behavioral disorders: diagnostic criteria for research. Madrid: Meditor.

Wolfe, V., Gentile, C., Michienzi, T., y Sas, L. (1991). The Children's Impact of Traumatic Events Scale: A measure of post-sexual-abuse PTSD symptoms. Behavioral Assessment, 13, 359-383.

Wolfe, V.V., Gentile, C., Michienzi, T.T., Sas, L., y Wolfe, D.A. (1991). The Children's Impact of Traumatic Events Scale: A measure of postsexual-abuse PTSD symptoms. Behavioral Assessment, 13, 359-383.

Zanarini, M.C (Ed.). (1997). Role of sexual abuse in the etiology of borderline personality disorder. Washington, DC: American Psychiatric Press.

Zimmerman, M., y Mattia, J.I. (1999). Is posttraumatic stress disorders underdiagnosed in routine clinical settings?. The Journal of Nervous and Mental Disease, 187, 420-428.

Zlotnick, C., y Pearlsten, T. (1997). Validation of the Structured Interview for Disorders of Extreme Stress. Comprehensive Psychiatry, 38, 243-247.

Zubizarreta, I., Sarasua, B., Echeburúa, E., Corral, P., Sauca, D., y Esparanza, I. (1994). Consecuencias psicológicas del maltrato doméstico. En E. Echeburúa (Ed.), Personalidades violentas. Madrid: Pirámide. 